\title{
DIRECTIONAL THRESHOLDING ALGORITHM FOR GRAY SCALE IMAGE SEGMENTATION
}

\author{
Dr Reka R, \\ Department of Computer Science and Engineering, \\ Annai Mathammal Sheela Engineering College, \\ Namakkal, Tamil Nadu, India \\ rekasatheesh@gmail.com
}

\begin{abstract}
The main aim of the image segmentation is to change the representation of the image so that the boundaries and objects in an image can be easily observed. In this study, a novel algorithm is proposed for the image segmentation using gray scale images. The codebook algorithm is used in the proposed approach for optimal multidirectional thresholding approach. The background and foreground pixel values are stored in the codebook. It uses standard deviations along the four directions to search the background and foreground pixels iteratively. The misclassification error and Jaccard index are used to measure the system efficiency. The mean of misclassification error is $95.80 \%$ with standard deviation of 1.91 and the mean of Jaccard index is $92.36 \%$ with standard deviation of 5.6. These measures shows the efficacy of the proposed system.
\end{abstract}

Keywords: Image segmentation, grayscale image, codebook, directional thresholding, misclassification error, Jaccard index.

\section{INTRODUCTION}

Adaptive gamma correction and threshold segmentation based image enhancement is discussed in [1]. The input images are segmented by using Otsu algorithm for multiple thresholding. The quality of the image is identified by average gray gradient method. Binary coded ant colony algorithm based image thresholding is discussed in [2]. The parameters are initialized and threshold selection is made for ant colony algorithm. The thresholding based ant colony algorithm is used for classification.

Recognition and segmentation of traffic sign in scene images is discussed in [3]. Initially, the color distance is computed. Then, the binary based threshold method is used for segmentation. Dynamic thresholding based on binary particle swarm optimization algorithm is discussed in [4]. The adaptive histogram method and thresholding segmentation is used. The thresholding value has the adequate number of thresholds with significant amount.

Document image binarization for threshold correction and ruled-line extraction is discussed in [5]. The input document image is preprocessed by converting the image into gray scale. Initial thresholding is used for the segmentation. The background of the image is determined. Then the threshold correction is also done to obtain binary image. Image segmentation based on wavelet domain binary partition is discussed in [6]. The wavelet transform based image features are extracted. Binary partition tree method is used for segmentation.

Threshold segmentation based road marking extraction is discussed in [7]. The road marking search is reduced by using inverse perspective mapping. The road image is segmented by local adaptive threshold and canny edge detection method. The geometric features are used for extraction. Maximum mutual 
information based image thresholding is discussed in [8]. Multi scale gradient multiplication transform method is used for the decomposition of input images. The gray level images are segmented by thresholding method.

Capsule image segmentation using edge detection technique is discussed in [9]. Initially, the capsule images are enhanced and segmented by using neural network. Then the borders are traced and analyzed. Image segmentation based multilevel thresholding algorithm is discussed in [10]. The multilevel thresholding based on principal swarm optimization, kapur and Otsu methods are used for the segmentation.

Traffic scenes based segmentation method for image sequence is discussed in [11]. At first, the preprocessing step is made to remove high frequency noise in the image using low pass filtering. Then the image is analyzed by adaptive thresholding to obtain a binary image. The geometric descriptors are analyzed to identify the bright regions. Region distribution and edge detection based image segmentation is described in [12]. The seed selection algorithm is used for the histogram equalization method. Edges are detected by multi threshold and single threshold. Region merging and texture elimination are used for the segmentation.

Palm print image based segmentation based on Euclidean distance and adaptive threshold is discussed in [13]. The input palm print images are preprocessed by median filter to remove noise. The region of interest area is extracted by Euclidean distance. Global thresholding method is used for segmentation. Handwritten image segmentation based single thresholding method is discussed in [14]. The input handwritten images are segmented by thresholding method then the peak signal to noise ratio is used to predict the threshold value.

A novel method for gray scale image segmentation using multidirectional thresholding method is discussed. The organization of paper is as follows: Methods and materials of the proposed system are explained in section 2 . The experimental results and discussion of gray scale image segmentation is explained in section 3. Finally, conclusion is given in the last section.

\section{METHODS AND MATERIALS}

Segmentation is one of the major tasks in digital image processing and analysis. The purpose of segmentation is to divide an image into regions which are uniform and homogeneous with respect to some characteristics such as gray level or texture. Segmentation can be critical for subsequent analysis and scene description. The optimal thresholding is also used in empty vehicle redistribution [15] and wheel set online measurement [16]. In this study, a novel algorithm for gray scale image segmentation is proposed. A codebook is organized in the proposed approach to store the foreground and background pixel values.

Initially the code book does not contain any foreground and background pixels. The proposed algorithm starts with finding a gray value $(G)$ within the image that has more occurrence than other gray values in that image. This gray value is inserted into the foreground list of codebook at first. Usually the edges are aligned along four directions D1, D2, D3 and D4 as shown in Figure 1. The standard deviation of a set of data points shows how much dissimilarity or distribution from the mean exists. A low standard deviation indicates closeness of the data points to the mean and a high value indicates the data points are spread over a large range of values. This property can be used to calculate an optimal threshold for segmentation of gray scale images. 


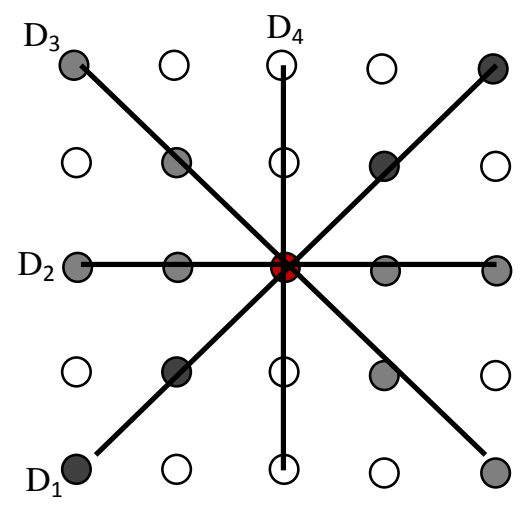

Fig. 1 Alignment of edges in four directions D1, D2, D3 and D4

To separate the pixels into foreground or background, the standard deviation along the four directions centered on $G$ need to be computed. The largest standard deviation along a direction indicates the large intensity variation among them; hence the gray values other than $G$ in that direction are inserted into the background list. The gray values in other directions are inserted into foreground list. As the foreground list is updated, this process is repeated for all the pixels in the foreground list. Then the threshold is calculated as the mean of the minimum and maximum intensity in the foreground list. To analyze the performance of the proposed thresholding technique, the segmentation accuracy is compared with other state of art techniques such as Otsu and iterative thresholding.

\section{RESULTS AND DISCUSSION}

The performance of the system is evaluated by 10 images in an image set having similar and dissimilar gray level histogram characteristics which is different from uni-model to multi-modal. The Jaccard index and misclassification error are computed as performance metrics.

The misclassification error is the Cartesian number of sets given in an image pixel. Misclassification error is also used in adjustable entropy [17]. The value of dissimilar images is 0 and the value of similar images is 100 . The misclassification error is defined by,

$$
\text { Misclassif ication } \operatorname{Error}(\delta)=\frac{\left|I_{0} \cap I_{T}\right|}{\left|I_{0}\right|} * 100
$$

where $I_{0}, I_{T}$ are resultant and gold standard image respectively. The similarity measure also made by Jaccard similarity coefficient, it is frequently used for binary data. The Jaccard index is also used in clustering coefficient [18]. The Jaccard index is given by,

$$
\text { Jaccard Index }\left(K_{i}\right)=\frac{\left|M_{i} \cap N_{i}\right|}{\left|M_{i} \cup N_{i}\right|} * 100
$$

where $N_{i}$ is the gold standard image, $M_{i}$ is the binary image and $K_{i}$ is the area of overlap calculated between the binary and gold standard image. Figure 2 shows 
the results of grayscale image segmentation based on multidirectional thresholding approach.

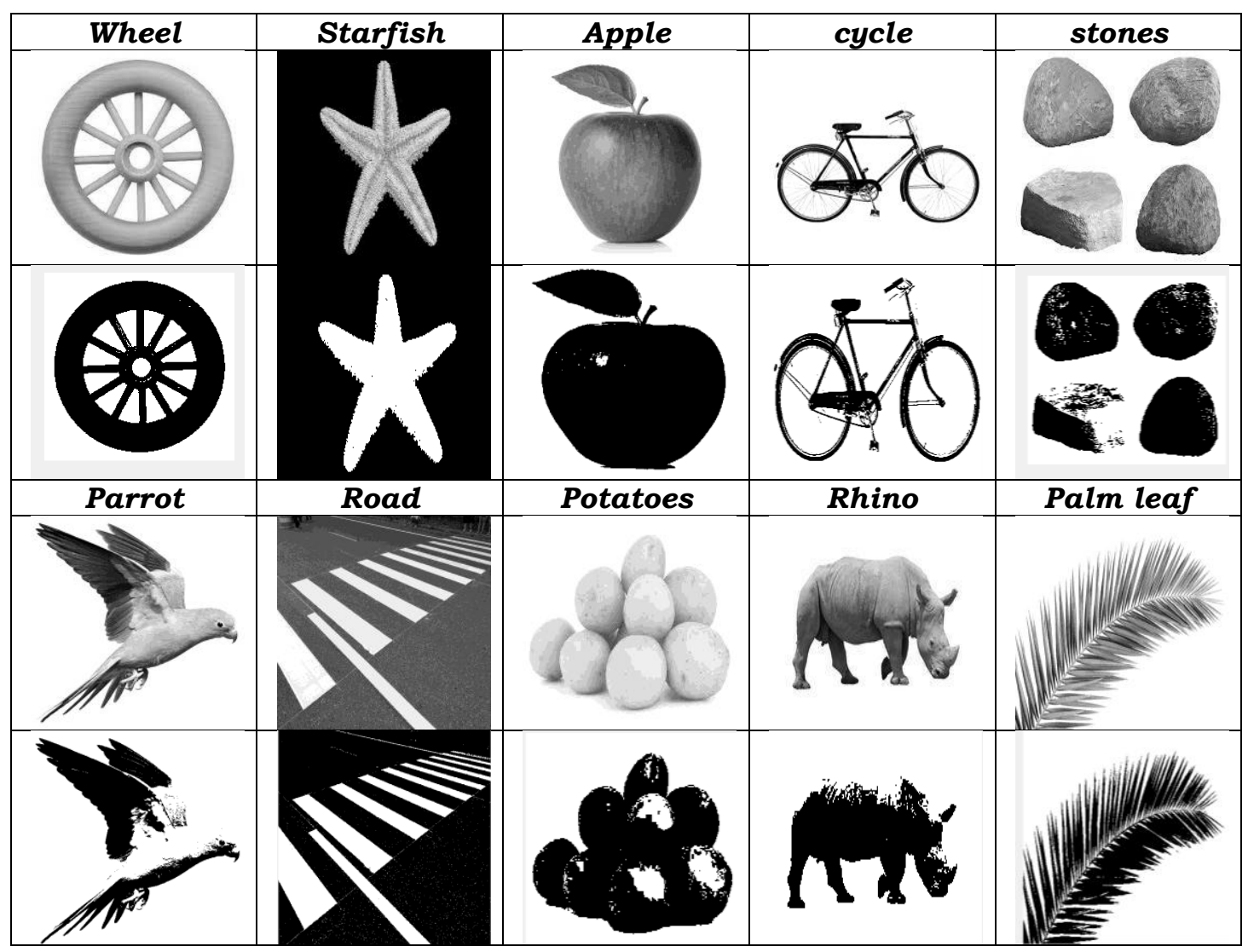

Fig. 3 Grayscale image segmentation based on multidirectional thresholding approach

The proposed system is compared with Tizhoosh method [19] and Otsu's [20] method in terms of misclassification error and jaccard index. Table 1 shows the misclassification error efficiency $\delta$ of Tizhoosh, Otsu's and proposed system.

TABLE 1 Misclassification error efficiency $\delta$ of Tizhoosh, Otsu's and proposed system (\%)

\begin{tabular}{|c|c|c|c|}
\hline Image set & Tizhoosh & Otsu & Proposed \\
\hline Wheel & 97.27 & 96.58 & 95.44 \\
\hline Starfish & 47.99 & 48.99 & 97.53 \\
\hline Apple & 96.61 & 94.17 & 95.84 \\
\hline cycle & 96.48 & 97.64 & 96.48 \\
\hline stones & 97.49 & 97.42 & 97.06 \\
\hline Parrot & 83.96 & 88.28 & 97.68 \\
\hline Road & 49.33 & 72.51 & 98.00 \\
\hline Potatoes & 95.15 & 96.79 & 94.83 \\
\hline Rhino & 94.97 & 90.92 & 91.69 \\
\hline Palm leaf & 96.53 & 94.95 & 93.5 \\
\hline
\end{tabular}


From the table 1 it is observed that the proposed system has the higher misclassification efficiency compare to Tizhoosh and Otsu methods. The mean and standard deviation of Tizhoosh, Otsu and proposed method is shown in Table 2.

TABLE 2 Mean and Standard deviation of Misclassification error efficiency

\begin{tabular}{|c|c|c|c|}
\hline Measure & Tizhoosh & Otsu & Proposed \\
\hline Mean (\%) & 85.57 & 87.82 & 95.80 \\
\hline Standard deviation & 18.83 & 14.89 & 1.91 \\
\hline
\end{tabular}

The Jaccard index efficiency is given in Table 3.

TABLE 3 Jaccard index of Tizhoosh, Otsu's and proposed system (\%)

\begin{tabular}{|c|c|c|c|}
\hline Image set & Tizhoosh & Otsu & Proposed \\
\hline Wheel & 96.55 & 95.19 & 93.01 \\
\hline Starfish & 75.24 & 85.73 & 84.14 \\
\hline Apple & 95.01 & 97.23 & 95.01 \\
\hline cycle & 95.27 & 90.62 & 93.77 \\
\hline stones & 97.00 & 96.85 & 96.14 \\
\hline Parrot & 31.32 & 32.22 & 97.07 \\
\hline Road & 32.53 & 57.37 & 98.00 \\
\hline Potatoes & 92.47 & 95.61 & 91.89 \\
\hline Rhino & 92.12 & 84.78 & 86.13 \\
\hline Palm leaf & 96.09 & 61.18 & 88.46 \\
\hline
\end{tabular}

From the above Table 3 it is observed that the proposed system has the higher Jaccard efficiency compare to Tizhoosh and Otsu methods. The mean and standard deviation of Tizhoosh, Otsu and proposed method is shown in Table 4.

TABLE 4 Mean and Standard deviation of Jaccard Index

\begin{tabular}{|c|c|c|c|}
\hline Measure & Tizhoosh & Otsu & Proposed \\
\hline Mean (\%) & 80.36 & 79.67 & 92.36 \\
\hline Standard deviation & 24.95 & 20.90 & 5.6 \\
\hline
\end{tabular}

\section{CONCLUSION}

A novel algorithm for grayscale image segmentation is presented in this study. The multidirectional thresholding method is used in the system for gray scale image segmentation. A codebook is used in this study in order to store the foreground and background pixel values. The misclassification error and Jaccard index are computed to analyze the system with other systems such as Tizhoosh and Otsu's method. The mean of misclassification error is $95.80 \%$ with standard deviation of 1.91 and the mean of Jaccard index is $92.36 \%$ with standard deviation of 5.6. Results show that the proposed approach produces higher efficiency with lower standard deviation in terms of misclassification error and Jaccard index that shows the accurate segmentation of the images. 


\section{REFERENCES}

[1]. J. Li, N. Wu, Z. Wang, J. Du, X. Fu, and C.C. Chang, "Enhancement of the Quality of Images Based on Multiple Threshold Segmentation and Adaptive Gamma Correction", International Conference on Intelligent Information Hiding and Multimedia Signal Processing, 2015, pp. 93-96.

[2]. Z. Ye, Z. Hu, H. Wang, and W. Liu, "A Image Thresholding Method Based on Binary Coded Ant Colony Algorithm", International Workshop on Intelligent Systems and Applications, 2010, pp. 1-4.

[3]. F. Qin, B. Fang, and H. Zhao, "Traffic sign segmentation and recognition in scene images", Chinese Conference on Pattern Recognition, 2010, pp. 1-5.

[4]. L. Djerou, H. Dehimi, N. Khelil, and M. Batouche, "Using the BPSO algorithm in image segmentation for dynamic thresholding", International on Conference on Bio-Inspired Computing, 2009, pp. 1-6.

[5]. H. Tanaka, "Threshold correction of document image binarization for ruled-line extraction", International Conference on Document Analysis and Recognition, 2009, pp. 541-545.

[6]. S. Ghanbari, J.C. Woods, H.R. Rabiee, and S.M. Lucas, "Wavelet domain binary partition trees for image segmentation", International Workshop on Content-Based Multimedia Indexing, 2008, pp. 302-307.

[7]. Z. Li, Z.X. Cai, J. Xie, and X.P. Ren, "Road markings extraction based on threshold segmentation", International Conference on Fuzzy Systems and Knowledge Discovery, 2012, pp. 1924-1928.

[8]. L. Fang, Y. Zou, F. Dong, S. Sun, and B. Lei, "Image thresholding based on maximum mutual information", International Congress on Image and Signal Processing, 2014, pp. 403-409.

[9]. M.J. Islam, S. Basalamah, M. Ahmadi, and M.A. Sid-Ahmed, "Capsule image segmentation in pharmaceutical applications using edge-based techniques", IEEE International Conference on Electro/Information Technology, 2011, pp. 1-5.

[10]. M. Dhieb, and M. Frikha, "A multilevel thresholding algorithm for image segmentation based on particle swarm optimization", International Conference of Computer Systems and Applications, 2016, pp. 1-7.

[11]. P. Sankaran, and V.K. Asari, "Adaptive thresholding based cell segmentation for cell-destruction activity verification", IEEE Applied Imagery and Pattern Recognition Workshop, 2006, pp. 14-14.

[12]. Y.R. Huang, and C.M. Kuo, "Image segmentation using edge detection and region distribution", International Congress on Image and Signal Processing, 2010, pp. 1410-1414.

[13]. S. Jun, "A fast self-adapt target image segmentation algorithm", Chinese Control Conference, 2008, pp. 500-504.

[14]. F. PirahanSiah, S.N.H.S. Abdullah, and S. Sahran, "Comparison single thresholding method for handwritten images segmentation", International Conference on Pattern Analysis and Intelligence Robotics, 2011, pp. 92-96.

[15]. D.P. Song, "Optimal threshold control of empty vehicle redistribution in two depot service systems", IEEE Transactions on Automatic Control, Vol. 50, No. 1, 2005, pp. 87-90.

[16]. W. Kaihua, and B. Tao, "Optimal threshold image segmentation method based on genetic algorithm in wheel set online measurement", International Conference on Measuring Technology and Mechatronics Automation, 2011, pp. 799-802. 
[17]. B. Sun, S.J. Song, C. Wu, and H. Zhang, "Adjustable entropy funtion method for misclassification minimization problems", International Conference on Machine Learning and Cybernetics, 2009, pp. 1556-1564.

[18]. A.K. Gupta, and N. Sardana, "Significance of clustering coefficient over jaccard index", International Conference on Contemporary Computing, 2015, pp. 463-466.

[19]. H. R. Tizhoosh, "Image thresholding using type II fuzzy sets," Pattern Recognition, Vol. 38, 2005, pp. 2363-2372.

[20]. N. Otsu, "A threshold selection method from gray level histograms," IEEE Transactions on System, Man, Cybernetics, Vol. SMC-9, 1979, pp. 62-66. 